\title{
BMJ Open The relationship of adiposity and mortality among people with diabetes in the US general population: a prospective cohort study
}

\author{
Andy Menke, ${ }^{1}$ Sarah S Casagrande, ${ }^{1}$ Catherine C Cowie ${ }^{2}$
}

To cite: Menke A, Casagrande SS, Cowie CC. The relationship of adiposity and mortality among people with diabetes in the US general population: a prospective cohort study. BMJ Open 2014;4:e005671. doi:10.1136/bmjopen-2014005671

- Prepublication history and additional material is available. To view please visit the journal (http://dx.doi.org/ 10.1136/bmjopen-2014005671).

Received 9 May 2014 Revised 10 October 2014 Accepted 30 October 2014

CrossMark

\begin{abstract}
${ }^{1}$ Social \& Scientific Systems, Inc, Silver Spring, Maryland, USA

${ }^{2}$ National Institute of Diabetes and Digestive and Kidney Diseases, National Institutes of Health, Bethesda, Maryland, USA
\end{abstract}

Correspondence to Dr Andy Menke; amenke@s-3.com

\section{ABSTRACT}

Objective: Several studies have found a U-shaped association between body mass index (BMI) and mortality in the general population. In similar studies among people with diabetes, the shape of the association is inconsistent. We investigated the relationship of BMI and waist circumference with mortality among people with diabetes.

Setting: The Third National Health and Nutrition Examination Survey (NHANES III) and the 1999-2004 NHANES Mortality Studies were designed to be representative of the US general population. Baseline data were collected in 1988-2004.

Participants: 2607 adults $\geq 20$ years of age with diabetes.

Primary Outcome Measure: Participants were followed through 31 December 2006 for mortality ( $\mathrm{n}=668$ deaths).

Results: Compared with people with a BMI $18.5-24.9 \mathrm{~kg} / \mathrm{m}^{2}$, the HRs $(95 \% \mathrm{Cl})$ of mortality were 0.85 (0.60 to 1.21 ) for $25-29.9 \mathrm{~kg} / \mathrm{m}^{2}, 0.87$ (0.57 to 1.33 ) for $30-34.9 \mathrm{~kg} / \mathrm{m}^{2}$ and 1.05 (0.72 to 1.53 ) for $\geq 35 \mathrm{~kg} / \mathrm{m}^{2}$ after adjustment for age, sex, race-ethnicity, smoking status, education, income and diabetes duration. Compared with people in the lowest sexspecific quartile of waist circumference, the adjusted HRs $(95 \% \mathrm{Cl})$ of mortality were $1.03(0.77$ to 1.37$)$ for the second quartile, 1.02 ( 0.73 to 1.42$)$ for the third quartile and 1.12 ( 0.77 to 1.61$)$ for the highest quartile of waist circumference. When modelled as a restricted quadratic spline with knots at the 10th, 50th and 90th centiles, $\mathrm{BMI}$ and waist circumference were not associated with mortality. Several sensitivity analyses were conducted and most found no significant association between measures of adiposity and mortality, but there were significant results suggesting a U-shaped association among people in the highest tertile of glycated haemoglobin $(\geq 7.1 \%)$, and there was an inverse association between $\mathrm{BMI}$ and mortality among people 20-44 years of age.

Conclusions: In a nationally representative sample of the non-institutionalised US population with diabetes, $\mathrm{BMI}$ and waist circumference were not associated with risk of mortality.

\section{Strengths and limitations of this study}

- This study used multiple biomarkers of adiposity to investigate the association between adiposity and mortality among people with diabetes. Body mass index and waist circumference were measured in participants by trained technicians using standardised protocols.

- Participants included people with diagnosed as well as undiagnosed diabetes from a large nationally representative sample.

- About $60 \%$ of the study participants were previously diagnosed with diabetes. Weight gain or loss may have occurred during the course of diabetes as a result of changes in lifestyle, medication use or diabetes disease progression.

- Mortality is the only outcome data we have for the participants since they were not actively followed for comorbidity. Therefore, our results cannot be generalised to non-fatal events or other diabetes complications.

\section{INTRODUCTION}

Several studies have found a U-shaped association between body mass index (BMI) and mortality in the general population, with underweight and obese people having a higher risk of mortality than those of normal weight. ${ }^{1}{ }^{2}$ Conversely, among people with certain chronic diseases, such as heart disease and kidney disease, studies suggest an obesity paradox may occur, whereby the risk of mortality is lower for obese than nonobese people. ${ }^{3-7}$ However, previous studies on the relationship between obesity and mortality among those with diabetes have been inconsistent. Large studies of people with diabetes from Scotland ${ }^{8}$ and Ukraine ${ }^{9}$ found a U-shaped association with the lowest risk of mortality in the range of $25-35 \mathrm{~kg} / \mathrm{m}^{2}$. Among diabetic participants of the Nurses' Health Study and Health Professionals' 
Follow-up Study, BMI had a J-shaped or direct linear relationship with mortality depending on smoking status. ${ }^{10}$ In the National Health Interview Survey ${ }^{11}$ and in a pooled analysis of five cohort studies, ${ }^{12}$ participants with diabetes who were overweight or obese had a lower risk of mortality than participants with diabetes who were of normal weight. Several other studies failed to find an association between BMI and mortality among people with diabetes. ${ }^{13-16}$ Few studies have examined the relationship between other measures of adiposity and mortality among people with diabetes. One study found that waist circumference was positively associated with risk of mortality. ${ }^{12}$

The purpose of our analysis was to characterise the relationship of BMI and waist circumference with mortality among people with diabetes. To do so, we analysed data from diabetic participants of the Third National Health and Nutrition Examination Survey (NHANES III), and the 1999-2004 NHANES Mortality Studies, cohort studies based on nationally representative samples of US adults in which baseline data, including BMI and waist circumference, were collected in 1988-2004, and participants were followed for mortality through 31 December 2006.

\section{METHODS}

Study population

NHANES III and the 1999-2004 NHANES were stratified, multistage probability samples of the non-institutionalised, civilian US population that were conducted by the National Center for Health Statistics of the Centers for Disease Control and Prevention (CDC). ${ }^{17}{ }^{18}$ We used a prospective cohort design to evaluate the association of BMI and waist circumference measured in adult participants with diabetes in 1988-2004 with mortality determined through 31 December 2006. Baseline data collected consisted of an in-home interview and a subsequent visit to a mobile examination centre. There were 2109 participants in NHANES III and 1841 participants in 1999-2004 NHANES $\geq 20$ years of age with diabetes, which we defined as a self-reported previous diagnosis, or previously undiagnosed diabetes based on the American Diabetes Association ${ }^{19}$ criteria of a glycated haemoglobin (HbAlc) $\geq 6.5 \%$, a fasting plasma glucose $\geq 126 \mathrm{mg} / \mathrm{dL}$ or a $2 \mathrm{~h}$ plasma glucose (following a $75 \mathrm{~g}$ oral glucose tolerance test) $\geq 200 \mathrm{mg} / \mathrm{dL}$. We excluded participants with a history of cardiovascular disease $(\mathrm{n}=846)$ or cancer $(n=235)$, pregnant women $(n=22)$, participants with likely type 1 diabetes $(n=38$; defined as having all 3 of the following criteria: previous diagnosis before 30 years of age, current insulin use and first insulin use within 1 year of diagnosis), participants with $\mathrm{BMI}<18.5 \mathrm{~kg} / \mathrm{m}^{2}(\mathrm{n}=15)$, participants who died in the first 2 years of follow-up $(n=129)$ and participants missing BMI and waist circumference data $(n=58)$. After these exclusions, we included a total of 2607 NHANES participants in our analysis. All participants gave written informed consent.

\section{Baseline data collection}

During the NHANES in-home interview, a standardised questionnaire was used to collect demographic information on age, race-ethnicity and sex. Additional information collected included education, income, smoking status, diabetes duration and use of antihypertensive medication.

Participants had their blood pressure measured up to six times and all blood pressure measurements for each participant were averaged. Hypertension was defined as systolic blood pressure $\geq 140 \mathrm{~mm} \mathrm{Hg}$, or diastolic blood pressure $\geq 90 \mathrm{~mm} \mathrm{Hg}$, or current use of antihypertensive medication. During the examination, a blood specimen was drawn from participants' antecubital vein by trained phlebotomists using a standardised protocol. Total serum cholesterol was measured enzymatically. ${ }^{17}$ is

During the examination, weight was measured using a digital scale to the nearest $0.01 \mathrm{~kg}$ and height was measured using a stadiometer to the nearest $0.1 \mathrm{~cm}$. BMI was calculated as weight in kilograms divided by height in metres squared. Waist circumference was measured at the iliac crest to the nearest $0.1 \mathrm{~cm}$.

\section{Mortality follow-up}

Study participants were passively followed for mortality through 31 December 2006 via linkage to the National Death Index. Participants were linked using probabilistic matching based on 12 identifiers (eg, Social Security number, sex, date of birth) to determine vital status and cause of death. To validate the probabilistic matching methodology, identical matching methodology was applied to the NHANES I Epidemiological Follow-up Study (which included active follow-up) and $96.1 \%$ of deceased participants and $99.4 \%$ of living participants were correctly classified. ${ }^{20}$

Cause of death was determined using The International Statistical Classification of Diseases and Related Health Problems (ICD), Ninth Revision, for deaths between 1988 and 1998 (codes 390-434 and 436-459), and ICD, Tenth Revision, for deaths between 1999 and 2006 (codes I00-I99). Cause-specific mortality was ascertained for cardiovascular disease (Ninth Revision codes 390-434 and 436-459; Tenth Revision codes I00-I99), cancer (Ninth Revision codes 140-208; Tenth Revision codes C00-C97) and respiratory issues (Ninth Revision codes 466, 480-487, 490-494, 496; Tenth Revision codes J10-J18, J20-J22, $\mathrm{J} 40-\mathrm{J} 47$ ) using the underlying cause of death. We were also interested in deaths in which diabetes played any role leading to death; to investigate this, diabetes mortality (Ninth Revision codes 250; Tenth Revision codes E10-E14) was defined using any cause of death listed on the death certificates (among the underlying cause and up to 20 contributing causes; the most common underlying causes of death among these participants were diabetes, heart disease, stroke and pneumonia).

\section{Statistical methods}

We calculated means and percentages of baseline levels of study characteristics by BMI category (18.5-24.9, 
25-29.9, 30-34.9 and $\geq 35 \mathrm{~kg} / \mathrm{m}^{2}$ ). For risk analyses, we excluded the first 2 years of follow-up to reduce the possibility that unidentified illness at baseline caused weight loss prior to causing death. We used Cox proportional hazards regression to estimate the HRs and $95 \%$ CIs for all-cause, cardiovascular, cancer, diabetes and respiratory mortality associated with BMI category. Next, we calculated HRs and 95\% CI for all-cause, cardiovascular, cancer, diabetes and respiratory mortality associated with sex-specific quartiles of the weighted waist circumference distributions; sex-specific cut points were used due to significantly different waist circumference distributions. To better characterise the shape of the association between BMI and all-cause mortality, we used restricted quadratic splines with knots at 25,30 and $35 \mathrm{~kg} / \mathrm{m}^{2}$. For waist circumference, we stratified by sex and used restricted quadratic splines with knots at the 10th, 50th and 90th centiles of the sex-specific waist circumference distributions (90.6, 105.9 and $124.8 \mathrm{~cm}$ for men; 84.5, 101.8 and $125.2 \mathrm{~cm}$ for women). For all analyses, initial models were unadjusted and subsequent models were adjusted for age (continuous), race-ethnicity (non-Hispanic white, non-Hispanic black, Mexican-American, other), sex, smoking (current $\geq 2$ pack/day, current 1-2 packs/ day, current $<1 \mathrm{pack} /$ day, former $\geq 2 \mathrm{pack} /$ day, former 1-2 packs/day, former <1 pack/day, never), education $(<$ high school, high school, >high school), income (<US\$20 000, $\geq$ US\$20 000) and diabetes duration (undiagnosed, $<5,5-9,10-14,15-19, \geq 20$ years).

We calculated the association between BMI category and all-cause mortality after stratifying by important covariates including diagnosed versus undiagnosed diabetes, HbAlc level, diabetes medication usage, hypertension status, cholesterol level, age and smoking category. Finally, we conducted a sensitivity analysis without excluding people with a history of cardiovascular disease or cancer, people with likely type 1 diabetes and the first 2 years of follow-up. Also, we calculated the risk of all-cause mortality associated with combined BMI and waist circumference, using previously described $^{21}$ combined risk categories (lowest risk: BMI $18.5-24.9 \mathrm{~kg} / \mathrm{m}^{2}$; increased risk: $25-29.9 \mathrm{~kg} / \mathrm{m}^{2}$ and low waist circumference; high risk: $25-29.9 \mathrm{~kg} / \mathrm{m}^{2}$ and high waist circumference or $30-34.9 \mathrm{~kg} / \mathrm{m}^{2}$ and low waist circumference; very high risk: $30-34.9 \mathrm{~kg} / \mathrm{m}^{2}$ and high waist circumference or $35-39.9 \mathrm{~kg} / \mathrm{m}^{2}$ and low waist circumference; extremely high risk: BMI $\geq 40 \mathrm{~kg} / \mathrm{m}^{2}$; high waist circumference defined as $>102 \mathrm{~cm}$ for men and $>88 \mathrm{~cm}$ for women).

Data were analysed using SUDAAN software (V.10; Research Triangle Institute, Research Triangle Park, North Carolina, USA) to account for the complex sampling design used in NHANES III.

\section{RESULTS}

Baseline characteristics

At baseline, $59.7 \%$ of participants had previously diagnosed diabetes and $40.3 \%$ had undiagnosed diabetes.
Among participants, 23\% had a BMI of 18.5$24.9 \mathrm{~kg} / \mathrm{m}^{2}, 35 \%$ had $25-29.9,25 \%$ had $30-34.9$ and $17 \% \mathrm{had} \geq 35 \mathrm{~kg} / \mathrm{m}^{2}$. Compared to participants with a lower BMI, those with a higher BMI were younger and more likely to be women, to be non-Hispanic black, to be never smokers, to be in the lowest category of diabetes duration, to take oral medications (without insulin), to have hypertension and to have a larger waist circumference; they were less likely to be in the lowest tertile of HbA1c and less likely to take no diabetes medication (table 1). The correlation between BMI and waist circumference was 0.923 for men and 0.893 for women.

\section{BMI and mortality}

The mean follow-up for participants was 6.5 years (maximum 16 years), during which 668 participants died. The mortality rates (SE) were 41 (5.6), 32 (3.4), 26 (3.4) and 19 (2.8) per 1000 person-years for participants with a BMI of 18.5-24.9, 25-29.9, 30-34.9 and $\geq 35 \mathrm{~kg} / \mathrm{m}^{2}$, respectively (table 2 ). Compared to participants with a BMI of $18.5-24.9 \mathrm{~kg} / \mathrm{m}^{2}$, the unadjusted HRs $(95 \% \mathrm{CI})$ for all-cause mortality were 0.79 (0.58 to $1.07), 0.64(0.46$ to 0.90$)$ and $0.48(0.33$ to 0.69$)$ for a BMI of $25-29.9,30-34.9$ and $\geq 35 \mathrm{~kg} / \mathrm{m}^{2}$, respectively. After multivariable adjustment, the HRs $(95 \%$ CI) for all-cause mortality were 0.85 (0.60 to 1.21 ), 0.87 (0.57 to $1.33)$ and 1.05 (0.72 to 1.53) for a BMI of 25-29.9, $30-34.9^{2}$ and $\geq 35 \mathrm{~kg} / \mathrm{m}^{2}$, respectively. The multivariable adjusted relative hazard of all-cause mortality associated with BMI is shown in figure 1; the curve was a shallow U-shape, but there were no significant differences along the distribution of BMI. When stratified by sex, the association among men had a deeper $U$ shape in which men with a BMI of approximately $28-33 \mathrm{~kg} / \mathrm{m}^{2}$ had a significantly lower risk of mortality than those with a BMI of $25 \mathrm{~kg} / \mathrm{m}^{2}$ (online supplementary figure $\mathrm{S} 1$ ). There was no evidence of a $\mathrm{U}$-shaped association among women and no significant differences along the distribution of BMI (online supplementary figure S2). The HRs for cardiovascular, cancer, diabetes and respiratory mortality were not significantly associated with BMI category after multivariable adjustment (table 2).

Further analyses of the association between BMI and all-cause mortality after stratifying by previous diabetes diagnosis (online supplementary table S1), HbAlc level (online supplementary table S2), diabetes medication usage (online supplementary table S3), hypertension status (online supplementary table S4), cholesterol level (online supplementary table S5), age (online supplementary table S6) and smoking category (online supplementary table S7) resulted in only two significant associations after multivariable adjustment. Among participants in the higher tertile of HbAlc $(\geq 7.1 \%)$, those with a BMI of $25-29.9 \mathrm{~kg} / \mathrm{m}^{2}$ had a significantly lower risk of mortality than those with a BMI of 18.5$24.9 \mathrm{~kg} / \mathrm{m}^{2}$. Although there were very few deaths among participants 20-44 years of age, those with a BMI 
Table 1 Means or percentages (SEs) of baseline participant characteristics by body mass index category

\begin{tabular}{|c|c|c|c|c|c|}
\hline & $\begin{array}{l}18.5-24.9 \mathrm{~kg} / \mathrm{m}^{2} \\
\mathrm{~N}=394\end{array}$ & $\begin{array}{l}25-29.9 \mathrm{~kg} / \mathrm{m}^{2} \\
\mathrm{~N}=925\end{array}$ & $\begin{array}{l}30-34.9 \mathrm{~kg} / \mathrm{m}^{2} \\
\mathrm{~N}=659\end{array}$ & $\begin{array}{l}\geq 35 \mathrm{~kg} / \mathrm{m}^{2} \\
\mathrm{~N}=565\end{array}$ & p-Trend \\
\hline Age, years & $60.4(1.17)$ & $59.4(0.74)$ & $56.1(0.68)$ & $51.3(0.64)$ & $<0.01$ \\
\hline Non-Hispanic white, \% & $62.6(4.20)$ & $63.7(2.57)$ & $68.5(2.53)$ & $66.6(2.65)$ & 0.27 \\
\hline Non-Hispanic black, \% & $11.2(1.58)$ & $15.0(1.45)$ & $12.9(1.27)$ & $18.8(2.03)$ & $<0.01$ \\
\hline Mexican-American, \% & $11.7(2.59)$ & $12.5(1.73)$ & $13.9(2.02)$ & $9.6(1.49)$ & 0.29 \\
\hline Women, \% & $51.5(3.68)$ & $44.0(2.44)$ & $43.6(2.90)$ & $63.1(3.18)$ & $<0.01$ \\
\hline Current smoker, \% & $26.8(3.25)$ & $18.3(2.03)$ & $20.8(2.47)$ & $17.4(2.29)$ & 0.09 \\
\hline Former smoker, \% & $30.8(3.43)$ & $40.1(2.71)$ & 36.8 (3.09) & $31.5(3.16)$ & 0.35 \\
\hline Never smoker, \% & $42.4(3.99)$ & $41.6(2.53)$ & $42.4(2.83)$ & $51.1(2.85)$ & 0.01 \\
\hline$<$ High school education, \% & $32.8(3.41)$ & $38.2(2.53)$ & $35.4(2.78)$ & $35.5(2.76)$ & 0.97 \\
\hline High school education, \% & $26.5(3.72)$ & $29.4(2.91)$ & $31.4(2.38)$ & 32.7 (3.37) & 0.19 \\
\hline$>$ High school education, \% & $40.7(4.15)$ & $32.4(2.46)$ & $33.2(2.98)$ & $31.8(3.29)$ & 0.20 \\
\hline Income $<$ US $\$ 20000, \%$ & $41.6(4.08)$ & $39.1(2.22)$ & $31.8(2.71)$ & $36.9(2.78)$ & 0.22 \\
\hline Previously diagnosed diabetes, \% & $60.7(4.03)$ & $59.6(2.58)$ & $50.5(2.60)$ & $60.8(3.37)$ & 0.93 \\
\hline Undiagnosed diabetes, \% & $39.4(4.03)$ & $40.4(2.58)$ & $49.5(2.60)$ & $39.2(3.37)$ & 0.93 \\
\hline Diabetes duration*, years & $10.6(0.74)$ & $9.6(0.72)$ & $9.1(0.79)$ & $8.8(0.83)$ & 0.13 \\
\hline Duration $<5$ years, $\%$ & $24.3(3.16)$ & $27.3(2.16)$ & $22.3(2.33)$ & $32.0(3.00)$ & 0.04 \\
\hline Duration $5-9$ years, \% & $15.2(2.45)$ & $10.7(1.23)$ & $12.8(1.53)$ & $10.7(1.51)$ & 0.27 \\
\hline Duration $10-14$ years, $\%$ & $7.8(1.50)$ & $9.2(1.19)$ & $5.9(1.29)$ & $7.4(1.46)$ & 0.49 \\
\hline Duration $15-19$ years, \% & $3.5(1.07)^{*}$ & $5.7(1.08)$ & $4.3(1.01)$ & $2.9(1.04)^{*}$ & 0.24 \\
\hline Duration $\geq 20$ years, $\%$ & $10.0(1.61)$ & $6.7(1.13)$ & $5.2(1.13)$ & $7.8(1.49)$ & 0.61 \\
\hline No exercise, \% & $39.7(5.17)$ & 36.7 (4.04) & $36.5(4.73)$ & $44.6(4.73)$ & 0.39 \\
\hline Exercise 1-3 times/week, \% & $44.2(5.49)$ & $48.3(4.57)$ & $44.8(5.07)$ & $27.6(5.03)$ & 0.01 \\
\hline Exercise $\geq 3$ times/week, \% & $16.1(4.18)$ & $15.0(2.23)$ & $18.8(3.20)$ & $27.8(3.62)$ & 0.02 \\
\hline Caloriest, kcal & $1718(100.1)$ & $1855(65.0)$ & 1987 (87.6) & $2011(128.0)$ & 0.08 \\
\hline Calories from saturated fat $\dagger$, mean $\%$ & $10.5(0.37)$ & $11.1(0.26)$ & $11.5(0.52)$ & $12.2(0.48)$ & 0.01 \\
\hline Mean $\mathrm{HbA} 1 \mathrm{c}$, mean \% & $7.1(0.18)$ & $7.1(0.08)$ & $7.3(0.11)$ & $7.2(0.10)$ & 0.48 \\
\hline $\mathrm{HbA} 1 \mathrm{c}<5.9 \%, \%$ & $40.0(3.75)$ & $32.5(2.44)$ & $28.3(2.98)$ & $26.3(3.10)$ & $<0.01$ \\
\hline HbA1c 5.9-7.0\%, \% & $22.7(2.83)$ & $34.4(2.60)$ & $36.8(2.93)$ & $39.0(3.10)$ & $<0.01$ \\
\hline $\mathrm{HbA} 1 \mathrm{c} \geq 7.1 \%, \%$ & $37.3(3.42)$ & $33.1(2.35)$ & $34.9(2.71)$ & 34.7 (2.68) & 0.86 \\
\hline Not taking medication, \% & $61.8(3.49)$ & $57.1(2.28)$ & $60.5(2.90)$ & $51.6(3.14)$ & 0.05 \\
\hline Taking oral medications only, \% & $25.0(2.75)$ & $31.4(2.13)$ & $27.7(2.74)$ & 36.7 (2.94) & 0.01 \\
\hline Taking insulin, \% & $13.2(2.58)$ & $11.5(1.37)$ & $11.8(1.50)$ & $11.7(1.51)$ & 0.70 \\
\hline Hypertension, \% & $44.2(4.15)$ & 56.7 (2.39) & 57.7 (3.19) & $64.0(3.27)$ & $<0.01$ \\
\hline Total cholesterol, mg/dL & $218.7(6.06)$ & $217.5(2.48)$ & $215.6(3.08)$ & $210.1(3.20)$ & 0.08 \\
\hline Waist circumference, $\mathrm{cm}$ & $87.8(0.51)$ & $99.6(0.36)$ & $109.0(0.44)$ & $125.0(1.03)$ & $<0.01$ \\
\hline
\end{tabular}

$\geq 25 \mathrm{~kg} / \mathrm{m}^{2}$ had a significantly lower risk of mortality than those with a BMI of $18.5-24.9 \mathrm{~kg} / \mathrm{m}^{2}$. Interaction terms for all stratified analyses were not significant, except for analyses stratified by HbAlc $(p=0.003)$ in which the HRs for the highest tertile of HbAlc were lower than the HRs for the lower tertiles of HbAlc. In a sensitivity analysis including only never smokers without a previous diabetes diagnosis, BMI was not significantly associated with mortality (online supplementary table S8). Results were similar in a sensitivity analysis when people with a history of cardiovascular disease or cancer, people with likely type 1 diabetes, and the first 2 years of follow-up were included (online supplementary table S9). Finally, the results were similar when we additionally adjusted for exercise and dietary variables (calories consumed and per cent of calories from saturation fat; data not shown).

\section{Waist circumference and mortality}

The mortality rates (SE) were 29 (3.5), 31 (4.0), 28 (3.5) and 23 (3.0) per 1000 person-years for waist circumference quartiles 1, 2, 3 and 4, respectively (table 3 ). Compared with participants in the first quartile of waist circumference, the unadjusted HRs $(95 \%$ CI) for allcause mortality were 1.11 (0.86 to 1.43 ), 0.99 (0.71 to 1.39 ) and 0.84 (0.61 to 1.17 ) for quartiles 2,3 and 4, respectively (table 3). After multivariable adjustment, the HRs $(95 \% \mathrm{CI})$ for all-cause mortality were 1.03 (0.77 to 1.37$), 1.02$ (0.73 to 1.42$)$ and 1.12 (0.77 to 1.61) for quartiles 2,3 and 4, respectively. The multivariable adjusted relative hazard of all-cause mortality associated with waist circumference is shown in figure 2. For men (figure 2A) and women (figure 2B), there were no significant differences along the distribution of waist circumference. The HRs for cardiovascular, cancer, 
Table $2 \mathrm{HR}(95 \% \mathrm{Cl})$ of all-cause and cause-specific mortality associated with body mass index category

\begin{tabular}{|c|c|c|c|c|}
\hline & $18.5-24.9 \mathrm{~kg} / \mathrm{m}^{2}$ & $25-29.9 \mathrm{~kg} / \mathrm{m}^{2}$ & $30-34.9 \mathrm{~kg} / \mathrm{m}^{2}$ & $\geq 35 \mathrm{~kg} / \mathrm{m}^{2}$ \\
\hline \multicolumn{5}{|l|}{ All-cause mortality } \\
\hline Number of deaths & 151 & 267 & 151 & 99 \\
\hline \multicolumn{5}{|l|}{ HRs } \\
\hline Unadjusted & 1.00 & 0.79 (0.58 to 1.07$)$ & 0.64 (0.46 to 0.90$)$ & 0.48 (0.33 to 0.69$)$ \\
\hline Multivariable adjusted $1 \dagger$ & 1.00 & $0.75(0.53$ to 1.08$)$ & 0.76 (0.51 to 1.12$)$ & $0.85(0.56$ to 1.31$)$ \\
\hline Number of deaths & 66 & 102 & 57 & 34 \\
\hline Mortality rate $(\mathrm{SE})^{*}$ & $15(2.9)$ & $12(2.1)$ & $6(1.3)$ & $6(1.5)$ \\
\hline \multicolumn{5}{|l|}{ HRs } \\
\hline Unadjusted & 1.00 & $0.78(0.49$ to 1.24$)$ & $0.44(0.25$ to 0.75$)$ & $0.40(0.22$ to 0.74$)$ \\
\hline Multivariable adjusted $1 \dagger$ & 1.00 & $0.79(0.47$ to 1.32$)$ & 0.59 (0.31 to 1.10$)$ & $0.86(0.46$ to 1.63$)$ \\
\hline Multivariable adjusted $2 \ddagger$ & 1.00 & $0.96(0.56$ to 1.64$)$ & 0.76 (0.40 to 1.46$)$ & $1.06(0.56$ to 2.00$)$ \\
\hline \multicolumn{5}{|l|}{ HRs } \\
\hline Unadjusted & 1.00 & $1.50(0.52$ to 4.36$)$ & $1.52(0.52$ to 4.46$)$ & $0.57(0.14$ to 2.38$)$ \\
\hline Multivariable adjusted $1 \dagger$ & 1.00 & $1.41(0.47$ to 4.18$)$ & 1.74 (0.60 to 5.05$)$ & 0.98 (0.23 to 4.12$)$ \\
\hline Multivariable adjusted $2 \ddagger$ & 1.00 & $1.73(0.54$ to 5.60$)$ & 1.90 (0.61 to 5.90$)$ & 1.39 (0.32 to 5.99$)$ \\
\hline \multicolumn{5}{|l|}{ Diabetes mortality } \\
\hline Number of deaths & 63 & 107 & 56 & 31 \\
\hline Mortality rate $(\mathrm{SE})^{*}$ & $18(4.4)$ & $12(1.7)$ & 8 (1.3) & $5(1.3)$ \\
\hline \multicolumn{5}{|l|}{ HRs } \\
\hline Unadjusted & 1.00 & 0.65 (0.38 to 1.13$)$ & $0.43(0.25$ to 0.74$)$ & $0.28(0.13$ to 0.59$)$ \\
\hline Multivariable adjusted $1 \dagger$ & 1.00 & $0.64(0.36$ to 1.14$)$ & 0.50 (0.27 to 0.91$)$ & 0.43 (0.20 to 0.93$)$ \\
\hline Multivariable adjusted $2 \ddagger$ & 1.00 & $0.72(0.40$ to 1.30$)$ & 0.67 (0.37 to 1.21$)$ & $0.57(0.25$ to 1.30$)$ \\
\hline \multicolumn{5}{|l|}{ Respiratory mortality } \\
\hline
\end{tabular}

Figure 1 Adjusted relative hazard of all-cause mortality associated with body mass index (BMI). Grey shading represents 95\% Cl; tick marks indicate deaths; background histogram of $\mathrm{BMI}$ displayed on the right axis.

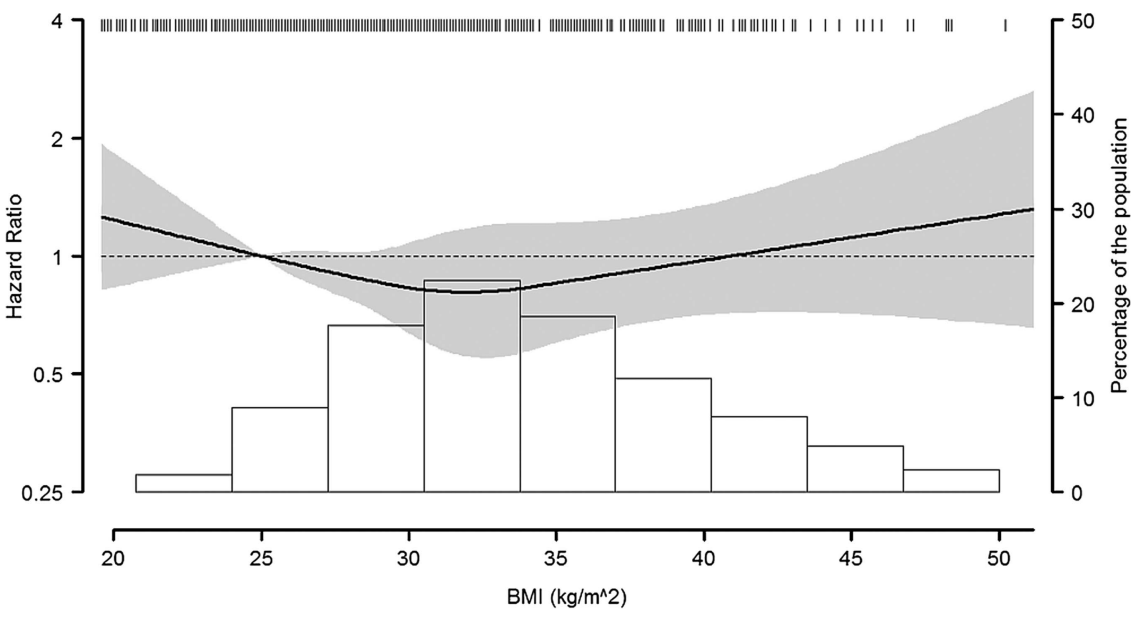


Table $3 \mathrm{HR}(95 \% \mathrm{Cl})$ of all-cause and cause-specific mortality associated with sex-specific ${ }^{*}$ quartile of waist circumference

\begin{tabular}{|c|c|c|c|c|}
\hline & Quartile 1 & Quartile 2 & Quartile 3 & Quartile 4 \\
\hline \multicolumn{5}{|l|}{ All-cause mortality } \\
\hline Number of deaths & 173 & 185 & 150 & 111 \\
\hline Mortality rate $(\mathrm{SE}) \dagger$ & $29(3.5)$ & $31(4.0)$ & $28(3.5)$ & $23(3.0)$ \\
\hline \multicolumn{5}{|l|}{ HRs } \\
\hline Unadjusted & 1.00 & 1.11 (0.86 to 1.43$)$ & 0.99 (0.71 to 1.39$)$ & 0.84 (0.61 to 1.17$)$ \\
\hline Multivariable adjusted $1 \ddagger$ & 1.00 & $0.92(0.69$ to 1.25$)$ & $0.87(0.61$ to 1.25$)$ & $1.04(0.69$ to 1.55$)$ \\
\hline Multivariable adjusted $2 \S$ & 1.00 & 1.03 (0.77 to 1.37$)$ & 1.02 (0.73 to 1.42$)$ & $1.12(0.77$ to 1.61$)$ \\
\hline \multicolumn{5}{|l|}{ Cardiovascular mortality } \\
\hline Number of deaths & 75 & 63 & 60 & 36 \\
\hline Mortality rate $(\mathrm{SE}) \dagger$ & $10(1.7)$ & $11(2.6)$ & $9(1.6)$ & $7(1.6)$ \\
\hline \multicolumn{5}{|l|}{ HRs } \\
\hline Unadjusted & 1.00 & $1.20(0.70$ to 2.07$)$ & 0.91 (0.57 to 1.45$)$ & $0.72(0.40$ to 1.31$)$ \\
\hline Multivariable adjusted $1 \ddagger$ & 1.00 & 1.00 (0.56 to 1.78$)$ & 0.80 (0.49 to 1.33$)$ & 1.09 (0.61 to 1.97$)$ \\
\hline Multivariable adjusted $2 \S$ & 1.00 & $1.17(0.66$ to 2.10$)$ & $0.94(0.59$ to 1.50$)$ & $1.13(0.64$ to 2.00$)$ \\
\hline \multicolumn{5}{|l|}{ Cancer mortality } \\
\hline Number of deaths & 31 & 46 & 26 & 24 \\
\hline Mortality rate $(\mathrm{SE}) \dagger$ & $7(2.2)$ & $7(2.3)$ & $8(2.2)$ & $5(1.4)$ \\
\hline \multicolumn{5}{|l|}{ HRs } \\
\hline Unadjusted & 1.00 & 1.07 (0.46 to 2.47$)$ & 1.22 (0.58 to 2.55$)$ & $0.67(0.27$ to 1.65$)$ \\
\hline Multivariable adjusted $1 \ddagger$ & 1.00 & 0.89 (0.39 to 2.04$)$ & $1.04(0.48$ to 2.26$)$ & $0.78(0.33$ to 1.85$)$ \\
\hline Multivariable adjusted $2 \S$ & 1.00 & $0.92(0.44$ to 1.90$)$ & 1.27 (0.57 to 2.82$)$ & 0.84 (0.34 to 2.08$)$ \\
\hline \multicolumn{5}{|l|}{ Diabetes mortality } \\
\hline Number of deaths & 68 & 78 & 54 & 34 \\
\hline Mortality rate $(\mathrm{SE}) \dagger$ & $12(2.7)$ & $13(2.3)$ & $8(1.4)$ & $6(1.4)$ \\
\hline \multicolumn{5}{|l|}{ HRs } \\
\hline Unadjusted & 1.00 & 1.12 (0.62 to 2.00$)$ & 0.68 (0.37 to 1.25$)$ & $0.55(0.30$ to 1.00$)$ \\
\hline Multivariable adjusted $1 \ddagger$ & 1.00 & $0.90(0.49$ to 1.66$)$ & $0.58(0.31$ to 1.08$)$ & $0.64(0.34$ to 1.18$)$ \\
\hline Multivariable adjusted $2 \S$ & 1.00 & 1.14 (0.58 to 2.22$)$ & $0.81(0.41$ to 1.59$)$ & $0.78(0.42$ to 1.46$)$ \\
\hline \multicolumn{5}{|l|}{ Respiratory mortality } \\
\hline Number of deaths & 12 & 13 & 9 & 9 \\
\hline Mortality rate $(\mathrm{SE}) \dagger$ & $3(1.1)$ & $3(1.0)$ & $2(0.8)$ & $2(1.0)$ \\
\hline \multicolumn{5}{|l|}{ HRs } \\
\hline Unadjusted & 1.00 & $1.21(0.40$ to 3.62$)$ & $0.71(0.21$ to 2.39$)$ & $0.87(0.25$ to 3.00$)$ \\
\hline Multivariable adjusted $1 \ddagger$ & 1.00 & 1.03 (0.35 to 2.98$)$ & 0.63 (0.18 to 2.24$)$ & $1.15(0.35$ to 3.76$)$ \\
\hline Multivariable adjusted $2 \S$ & 1.00 & $0.76(0.22$ to 2.62$)$ & $0.72(0.18$ to 2.90$)$ & $0.99(0.28$ to 3.54$)$ \\
\hline
\end{tabular}

*For men: <98.0, 98.0-105.1, 105.2-113.8 and $\geq 113.9 \mathrm{~cm}$; for women: $<90.4,90.4-100.8,100.9-111.5$ and $\geq 111.6 \mathrm{~cm}$.

†Per 1000 person-years.

$\ddagger$ Adjusted for age, race-ethnicity and sex.

$\S$ Adjusted for age, race-ethnicity, sex, smoking status, education, income and diabetes duration.

diabetes and respiratory mortality were not significantly associated with quartile of waist circumference after multivariable adjustment (table 3). After stratifying by sex, quartiles of waist circumference were not significantly associated with mortality among men or women (online supplementary table S10). Using previously described combined BMI and waist circumference categories, higher levels of adiposity were not significantly associated with all-cause mortality (online supplementary table S11).

\section{DISCUSSION}

In contrast to the U-shaped association observed in general population studies, our population-based prospective study of people with diabetes at baseline found that BMI and waist circumference were not significantly associated with risk of mortality. In sensitivity analyses in which we stratify the analysis by important characteristics, there were significant results suggesting a U-shaped association between BMI and mortality among men and among people in the highest tertile of HbAlc $(\geq 7.1 \%)$; among people $20-44$ years of age, those with a healthy weight based on BMI had a higher risk of mortality than those with higher BMI. However, the majority of our sensitivity analyses found no significant association between measures of adiposity and mortality.

Our findings in the overall diabetes population showing no association between BMI and mortality are consistent with several previous studies including a study of 8334 people with diabetes ${ }^{14}$ and two other smaller studies ${ }^{13} 15$ that did not find an association between BMI and mortality among people with diabetes. Conversely, other studies investigating this relationship have had inconsistent results. In a study of medical records from over 100000 people with diabetes in Scotland, there was 
Figure 2 Adjusted relative hazard of all-cause mortality associated with waist circumference among men $(A)$ and women (B). Grey shading represents $95 \% \mathrm{Cl}$; tick marks indicate deaths; background histogram of waist circumference displayed on the right axis.
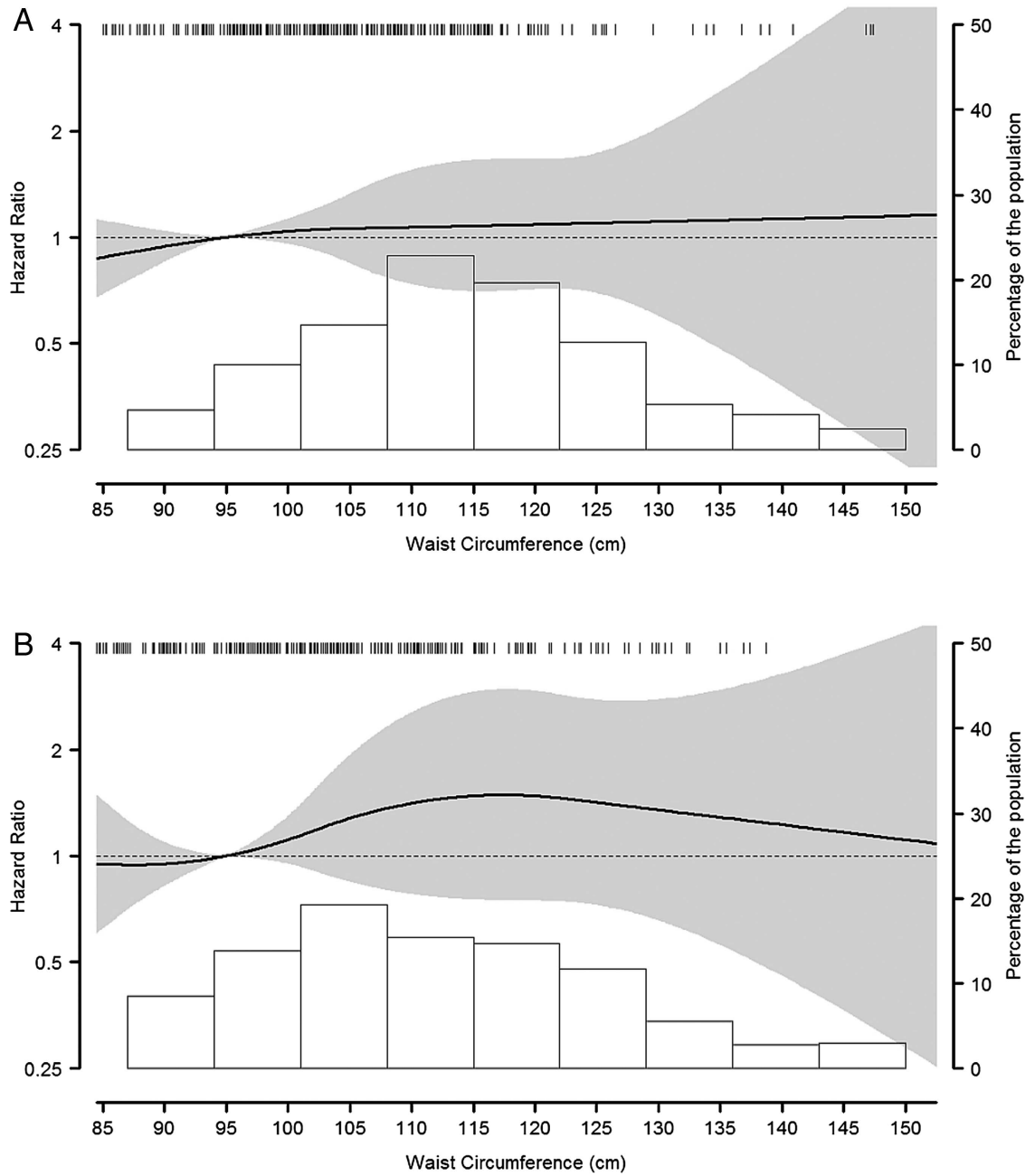

a U-shaped association and people with a BMI between 25 and $35 \mathrm{~kg} / \mathrm{m}^{2}$ had the lowest risk of mortality. ${ }^{8}$ Similarly, among over 89000 Ukrainian people in a population-based diabetes registry, there was a U-shaped association and people with a BMI between 25 and $30 \mathrm{~kg} / \mathrm{m}^{2}$ had the lowest risk of mortality. ${ }^{9}$ Among 8970 participants of the Nurses' Health Study and 2457 participants of the Health Professionals' Follow-up Study with diabetes, the shape of the BMI-mortality association depended on the smoking status of participants, with a J-shaped relationship among ever smokers and a positive linear relationship among never smokers. ${ }^{10}$ In a study of 13087 people in the Swedish National Diabetes Registry, BMI categories were positively related to risk of mortality. ${ }^{22}$ Among 4740 diabetic participants of the National Health Interview Survey, there was a monotonic decrease in risk of all-cause mortality associated with higher quintiles of self-reported BMI among never smokers and ever smokers. ${ }^{11}$ In a pooled analysis of five cohort studies including 2625 people with diabetes, ${ }^{12} \mathrm{BMI}$ was dichotomised and those $\geq 25 \mathrm{~kg} / \mathrm{m}^{2}$ had a lower risk of mortality than those with a BMI of $18.5-24.9 \mathrm{~kg} / \mathrm{m}^{2}$. These studies vary in terms of location, population, BMI categorisation, BMI assessment and timing of BMI measurement relative to diabetes diagnosis, but it is unclear why they are inconsistent.
The authors of a previous study suggested that collider bias may play a role in the inverse BMI-mortality association found in some previous studies of people with diabetes; they used NHANES data to investigate the issue, finding an inverse association between BMI and mortality only among smokers, an association that was attenuated and not significant after introducing restrictions aimed at reducing the intensity of reverse causal pathways. ${ }^{16}$ Our study similarly excluded participants to limit the impact of reverse causal pathways and we found no association between multiple measures of adiposity and mortality. The non-significant positive association among never smokers suggests the possibility that there are some residual biases in our analysis of the overall population and completely eliminating the biases may result in a positive association between adiposity and mortality.

There are other possible explanations for why the association between adiposity and mortality may differ in persons with diabetes compared with the general population. Since type 2 diabetes is an obesity-related disease, ${ }^{19}$ people with normal weight who develop diabetes may have additional risk factors for diabetes that also increase risk of mortality, or they may have a more aggressive pathophysiology. It is also possible that people with normal weight and diabetes are screened less vigorously for cardiovascular disease and cancer, offsetting 
any benefit of having less adipose tissue. It is important to note that our results do not provide any insight into whether gaining or losing weight affects risk of mortality among people with diabetes, because clinical characteristics were only obtained at baseline. ${ }^{23}$

Waist circumference is an important measure of adiposity, as it has been shown to be more strongly associated with obesity-related comorbidities than BMI. ${ }^{24-27}$ Muscle mass does not substantially affect waist circumference measurement, unlike BMI, which may be lower due to loss of lean body mass among the frail or among those with chronic ailments such as cardiovascular disease and cancer. ${ }^{28}$ We were only able to identify one previous study investigating the association between waist circumference and mortality among people with diabetes. In a pooled analysis of five cohort studies including 2625 participants, waist circumference modelled as a continuous variable was found to be positively associated with risk of mortality after multivariable adjustment. ${ }^{12}$ In contrast, waist circumference was not associated with mortality in our study.

In studies of adiposity and mortality among people with diabetes, the timing of when BMI or waist circumference is measured relative to diagnosis of diabetes may be important. In our study, about $60 \%$ of the participants were previously diagnosed with diabetes. Weight gain or loss may have occurred during the course of diabetes as a result of changes in lifestyle, medication use or diabetes disease progression. However, when stratified by previous diabetes diagnosis, the BMI-mortality association was similar and not significant for those previously diagnosed and those who were undiagnosed at baseline. The diagnostic criteria and treatment regimens commonly used in the USA may have changed since the baseline of our study, which may affect the generalisability of our results to people who currently have diabetes. Additionally, comorbid conditions that increase the risk of mortality and cause weight loss could make obesity appear to have a relatively lower mortality risk compared with normal weight individuals. In our study, we attempted to prevent this by excluding people with a history of cardiovascular disease or cancer as well as those with undiagnosed conditions who died during the first 2 years of follow-up; however, some participants may have had undiagnosed diseases at baseline, which affected their adiposity measurements and increased their risk of mortality more than 2 years after baseline. Another potential limitation is that the adiposity-mortality relationship may differ between people with type 1 and type 2 diabetes. We excluded participants with likely type 1 diabetes; although we may not have identified every case of type 1 diabetes, there is not likely to be enough unidentified cases to have a substantial impact on our results. Also, some of our participants may have had other forms of diabetes, such as latent autoimmune diabetes in adults, which we were not able to identify, but these forms of diabetes are not common. Another limitation was the limited power to detect associations in some analyses of cause-specific mortality and in some sensitivity analyses in which we stratified results. Finally, mortality is the only outcome data we have for NHANES participants since they were not actively followed for comorbidity. Therefore, our results cannot be generalised to non-fatal events or other diabetes complications.

Despite our limitations, our study maintained a number of strengths. Our study included a large nationally representative sample of the non-institutionalised population with diabetes in the USA, and we were able to include people with diagnosed and undiagnosed diabetes. Also, multiple markers of adiposity, including BMI and waist circumference, were measured by NHANES staff using rigorous study protocols with extensive quality control procedures that changed minimally over time. Our results were consistent in that we found similar results for both markers of adiposity.

In conclusion, measures of adiposity were generally not associated with mortality in a nationally representative sample of people with prevalent and incident diabetes. However, BMI had a U-shaped association with mortality among men when modelled as a spline.

Contributors AM developed the study concept and design, conducted the statistical analysis and wrote the manuscript. SSC and CCC guided the statistical analysis and revised the manuscript.

Funding This work was supported by a contract from the National Institute of Diabetes and Digestive and Kidney Diseases (GS10F0381L).

\section{Competing interests None.}

Ethics approval The protocols for NHANES III and the 1999-2004 NHANES were approved by the CDC Ethics Review Board.

Provenance and peer review Not commissioned; externally peer reviewed.

Data sharing statement No additional data are available.

Open Access This is an Open Access article distributed in accordance with the Creative Commons Attribution Non Commercial (CC BY-NC 4.0) license, which permits others to distribute, remix, adapt, build upon this work noncommercially, and license their derivative works on different terms, provided the original work is properly cited and the use is non-commercial. See: http:// creativecommons.org/licenses/by-nc/4.0/

\section{REFERENCES}

1. Berrington de Gonzalez A, Hartge P, Cerhan JR, et al. Body-mass index and mortality among 1.46 million white adults. $N$ Engl $J$ Med 2010;363:2211-19.

2. Flegal $\mathrm{KM}$, Kit BK, Orpana $\mathrm{H}$, et al. Association of all-cause mortality with overweight and obesity using standard body mass index categories: a systematic review and meta-analysis. JAMA 2013;309:71-82.

3. Curtis JP, Selter JG, Wang Y, et al. The obesity paradox: body mass index and outcomes in patients with heart failure. Arch Intern Med 2005;165:55-61.

4. Galal W, van Gestel YR, Hoeks SE, et al. The obesity paradox in patients with peripheral arterial disease. Chest 2008;134:925-30.

5. Lavie CJ, Milani RV, Ventura HO, et al. Body composition and heart failure prevalence and prognosis: getting to the fat of the matter in the "obesity paradox". Mayo Clin Proc 2010;85:605-8.

6. Schmidt D, Salahudeen A. The obesity-survival paradox in hemodialysis patients: why do overweight hemodialysis patients live longer? Nutr Clin Pract 2007;22:11-15.

7. Uretsky S, Messerli FH, Bangalore S, et al. Obesity paradox in patients with hypertension and coronary artery disease. Am J Med 2007;120:863-70

8. Logue J, Walker JJ, Leese G, et al. Association between BMI measured within a year after diagnosis of type 2 diabetes and mortality. Diabetes Care 2013;36:887-93. 
9. Khalangot $\mathrm{M}$, Tronko M, Kravchenko $\mathrm{V}$, et al. Body mass index and the risk of total and cardiovascular mortality among patients with type 2 diabetes: a large prospective study in Ukraine. Heart 2009:95:454-60.

10. Tobias DK, Pan A, Jackson CL, et al. Body-mass index and mortality among adults with incident type 2 diabetes. N Engl J Med 2014;370:233-44.

11. Jackson $\mathrm{CL}$, Yeh HC, Szklo M, et al. Body-mass index and all-cause mortality in US adults with and without diabetes. J Gen Intern Med 2014;29:25-33.

12. Carnethon MR, De Chavez PJ, Biggs ML, et al. Association of weight status with mortality in adults with incident diabetes. JAMA 2012;308:581-90.

13. Knuiman MW, Welborn TA, Whittall DE. An analysis of excess mortality rates for persons with non-insulin-dependent diabetes mellitus in Western Australia using the Cox proportional hazards regression model. Am J Epidemiol 1992;135:638-48.

14. McEwen LN, Karter AJ, Waitzfelder BE, et al. Predictors of mortality over 8 years in type 2 diabetic patients: Translating Research Into Action for Diabetes (TRIAD). Diabetes Care 2012;35:1301-9.

15. Rosengren A, Welin L, Tsipogianni A, et al. Impact of cardiovascular risk factors on coronary heart disease and mortality among middle aged diabetic men: a general population study. BMJ 1989;299:1127-31.

16. Preston $\mathrm{SH}$, Stokes A. Obesity paradox: conditioning on disease enhances biases in estimating the mortality risks of obesity. Epidemiology 2014;25:454-61.

17. National Center for Health Statistics. Plan and operation of the third national health and nutrition examination survey, 1988-1994. Series 1: programs and collection procedures. Vital Health Stat 11994 ; (32):1-407.

18. Zipf G, Chiappa M, Porter KS, et al. National Health and Nutrition Examination Survey: plan and operations, 1999-2010. National Center for Health Statistics. Vital Health Stat 1 2013;(56):1-37.
19. American Diabetes Association. Diagnosis and classification of diabetes mellitus. Diabetes Care 2010;33(Suppl 1):S62-9.

20. National Center for Health Statistics. The third national health and nutrition examination survey linked mortality file: matching methodology. Hyattsville, MD: US Department of Health and Human Services, Centers for Disease Control and Prevention, 2006.

21. National Institutes of Health, National Heart, Lung, and Blood Institute. Clinical guidelines on the identification, evaluation, and treatment of overweight and obesity in adults: the evidence report. National Institutes of Health, 1998.

22. Eeg-Olofsson K, Cederholm J, Nilsson PM, et al. Risk of cardiovascular disease and mortality in overweight and obese patients with type 2 diabetes: an observational study in 13,087 patients. Diabetologia 2009;52:65-73.

23. Wing RR, Bolin P, Brancati FL, et al. Cardiovascular effects of intensive lifestyle intervention in type 2 diabetes. $N$ Engl J Med 2013;369:145-54

24. Foucan L, Hanley J, Deloumeaux J, et al. Body mass index (BMI) and waist circumference (WC) as screening tools for cardiovascular risk factors in Guadeloupean women. J Clin Epidemiol 2002:55:990-6.

25. Janssen I, Katzmarzyk PT, Ross R. Waist circumference and not body mass index explains obesity-related health risk. Am J Clin Nutr 2004;79:379-84.

26. Wei M, Gaskill SP, Haffner SM, et al. Waist circumference as the best predictor of noninsulin dependent diabetes mellitus (NIDDM) compared to body mass index, waist/hip ratio and other anthropometric measurements in Mexican Americans-a 7-year prospective study. Obes Res 1997:5:16-23.

27. Zhu S, Wang Z, Heshka S, et al. Waist circumference and obesity-associated risk factors among whites in the third National Health and Nutrition Examination Survey: clinical action thresholds. Am J Clin Nutr 2002;76:743-9.

28. Dorner TE, Rieder A. Obesity paradox in elderly patients with cardiovascular diseases. Int J Cardiol 2012;155:56-65. 